\title{
Les principes fondamentaux de la motivation au travail : Vers une amélioration de la productivité des établissements de l'enseignement supérieur marocains
}

\author{
TARHI Mohammed Amine
}

Département sciences économiques et de gestion à la FSJESAC Université Hassan II, Casablanca, Maroc

\begin{abstract}
Résumé : Il est communément admis aujourd'hui, que les établissements de l'enseignement supérieur sont devenus aussi soucieux de leur productivité et de leur efficacité pareillement aux autres organisations. C'est ainsi, qu'on constate généralement un grand écart entre ces établissements dans leur efficacité et efficience ainsi que dans leur productivité et rendement. Cette distinction réside même à l'intérieur de ces établissements, où on se retrouve devant une divergence de productivité que ce soit de la part du corps professoral et ou du corps administratif.

Cette variation de productivité est intimement liée à l'autoritarisme, et pratiques de gestion adoptées par les managers, ainsi qu'à la motivation et à la valorisation accordée aux collaborateurs en place.
\end{abstract}

Mots-clés : Motivation au travail, GRH, Productivité, Enseignement supérieur.

\section{INTRODUCTION}

Il existe très peu de recherches scientifiques qui se sont intéressées à l'impact des pratiques managériales sur la motivation des collaborateurs et la productivité au sein des organisations, d'au moins au sein des établissements de l'enseignement supérieur.

Plusieurs stratégies s'intéressent à appliquer des politiques qui visent dans son ensemble le perfectionnement des services, et l'amélioration des méthodes et des processus de production, mais sont rares les stratégies qui se focalisent sur l'Homme, et qui accordent plus d'attention à son bien-être et à sa satisfaction au travail.

À ce propos, nous nous sommes focalisés sur ce champ d'application pour mener une recherche dans ce domaine. En vue de comprendre le fonctionnement organisationnel, et de découvrir les meilleures pratiques de management qui mènent à un rendement supérieur et à une satisfaction meilleure.

Pour cela, nous avons mené une étude auprès de treize (13) établissements de l'enseignement supérieur marocains, qui relèvent tous de l'université HASSAN II de Casablanca.

En termes de scientificité de cette recherche, nous avons cherché à combiner les deux méthodes de collecte et d'analyse de données; à savoir: la méthode quantitative et la méthode qualitative pour assurer un minimum d'interprétation qualitative des relations entre les variables et des corrélations existantes. Comme nous avons limité notre échantillon représentatif de la population étudiée à $30 \%$ de l'effectif global, suivant l'incapacité d'entretenir tous les collaborateurs, ou comme stipulaient Miles \& Huberman (2003) «vous ne pourriez jamais étudier toutes les personnes dans tous les lieux possibles et examiner leurs actions»

L'un des concepts de base de cette recherche est que, quel que soit le domaine de spécialité des établissements étudiés, il existe, certes, des principes spécifiques et particuliers de la motivation au travail propres à chaque établissement, mais on retrouve généralement des principes communs et fondamentaux qui s'appliqueront à tous types d'organisation. 
Donc, notre intérêt majeur est de détecter et de déceler l'ensemble de ces principes, pour faire sortir des données scientifiquement valables, qui vont permettre de définir les principes généraux d'une bonne efficacité organisationnelle et d'une meilleure satisfaction au travail, en se basant principalement sur le développement des principes de motivation et de leurs mises en application.

Ainsi, durant tout le processus de cette recherche nous nous sommes basés sur deux principaux critères qu'on a retenus pour procéder à l'évaluation de l'efficacité administrative de ces établissements, il s'agit de :

- La productivité : qui tient compte principalement :

○ Du côté des enseignants : du nombre d'heures d'absence, de la cadence et du niveau de production scientifique, du nombre d'heures effectif de travail (heurs d'enseignement et heures d'encadrement), de l'implication dans la sphère organisationnelle, et d'autre mesure similaire.

○ Du côté du staff administratif : du nombre d'heures de travail, du taux d'absentéisme, du degré de réalisation des tâches et missions, et d'autre mesure similaire.

- La satisfaction au travail : qui correspond aux différentes formes de satisfaction ressenties par le corps professoral et le staff administratif.

L'évaluation de l'efficacité de ces treize établissements, en se basant sur les deux critères sus-indiqués, nous a permis de procéder dans un premier temps à la classification des corps professoral et administratif appartenant à ces établissements entre les plus productifs et les moins productifs. Par la suite, nous sommes parvenus à distinguer entre les établissements les plus productifs de ceux les moins productifs.

Nous présenterons dans ce qui suit quelques résultats obtenus de cette étude, comme nous relatons les principales conclusions.

\section{LES DÉTERMINANTS MAJEURS DU NIVEAU DE LA PRODUCTIVITÉ :}

Tout d'abord, il importe de préciser que la mesurer de la productivité des administratifs et des techniciens relevant des établissements de l'enseignement supérieur reste beaucoup plus simple et beaucoup plus facile que celle des enseignants-chercheurs dont l'activité demeure relativement complexe. Complexe dans la mesure où l'activité des enseignants-chercheurs est multidimensionnelle et donc reste difficilement mesurable, en effet, la publication d'article de recherche qui est la plus simple dimension reste jusqu'à la date d'aujourd'hui difficile à mesurer. Aussi pour pouvoir comparer la productivité du staff administratif et du corps professoral des établissements de l'enseignement supérieur marocains, il faut impérativement raisonner "toutes choses égales par ailleurs" cela veut dire que tous les EES marocain sont sur un pied d'égalité du côté des moyens, des outils, et des techniques misent à leurs dispositions, chose qui reste loin d'être justifiée.

Nous prétendons ici de présenter certains facteurs que nous jugeons aptes d'influencer positivement ou négativement le niveau de productivité, et qu'après examen de nos résultats de recherche ces derniers se révèlent dérisoire et insignifiant.

\section{A. La satisfaction au travail}

Nous avons découvert, qu'il existe une très faible corrélation entre le niveau de la productivité et la satisfaction des enseignants-chercheurs et du staff administratif à l'égard de leurs établissements (au sein du même établissement et entre tous les établissements). Donc le développement d'une attitude favorable à l'égard de l'établissement ne se traduit nullement pas par une meilleure productivité. La figure $\mathrm{n}^{\circ} 1$ décrit clairement ce constat et dévoile presque un pourcentage moyen de satisfaction des collaborateurs vis-à-vis de leur établissement indépendamment du niveau de leur productivité. 
TARHI Mohammed Amine : Les principes fondamentaux de la motivation au travail : Vers une amélioration ...

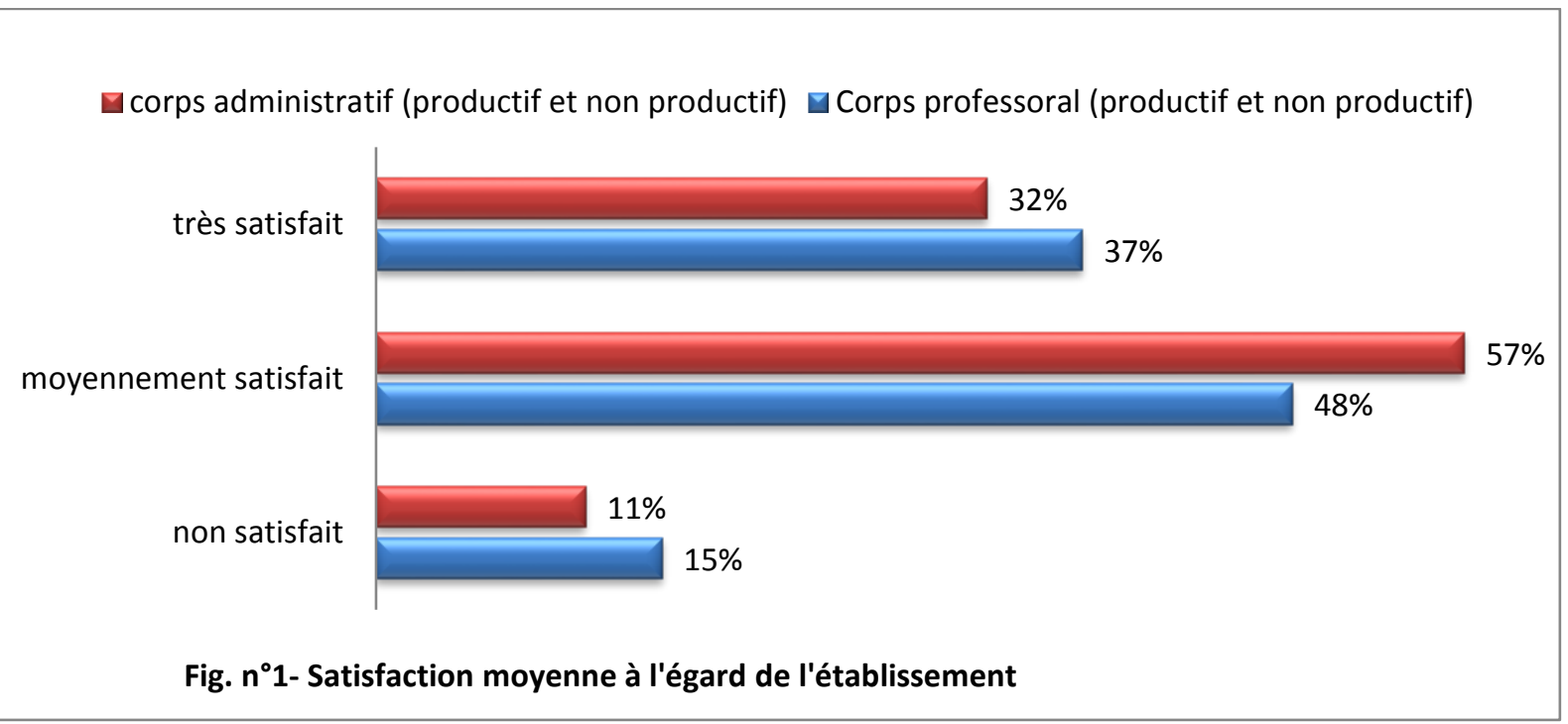

Par contre, nous avons vérifié qu'une meilleure satisfaction des collaborateurs à l'égard de leur établissement, et du travail en lui-même conduit à une diminution du taux d'absentéisme, au développement de l'esprit d'appartenance, et surtout à attirer des nouvelles compétences.

\section{B. Les événements festifs}

Un deuxième constat, tel que présente la figure $n^{\circ} 2$, et qui traite une autre variable relative aux événements festifs, montre clairement que ce dernier ne dévoile aucune corrélation positive avec la productivité. Dans la mesure où nous avons découvert que dans un certain nombre de situations, il existait une corrélation négative entre le taux de participation du corps professoral et administratif aux événements festifs et leur productivité. Ce sont les groupes à faible productivité qui participent souvent à ces événements que les groupes à forte productivité.

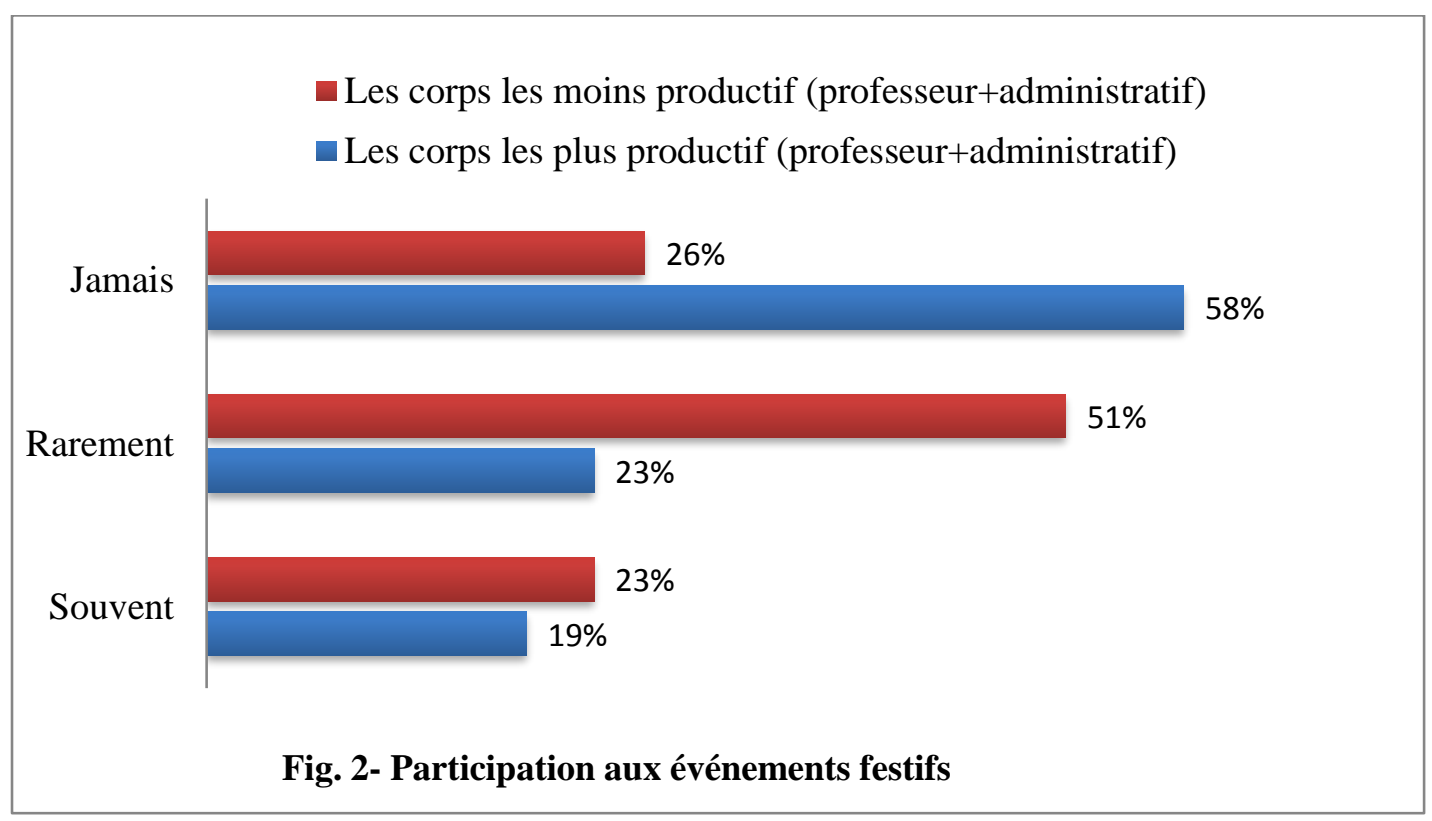




\section{Les styles de commandement}

Contrairement à ce qui a été constaté plus haut, nous avons relevé une forte corrélation entre le style de management et le niveau de productivité. Ainsi, si on considère le collaborateur comme une machine de production et qu'on a limité son rôle à l'exécution des tâches assignées sans lui donner une certaine marge de manœuvre, ce dernier se placera sans doute parmi les faibles producteurs. Par contre, si on s'intéresse davantage à son bien-être, à sa satisfaction et à son avenir, une meilleure productivité se réalisera. La figure n³ illustre avec précision ce constat.

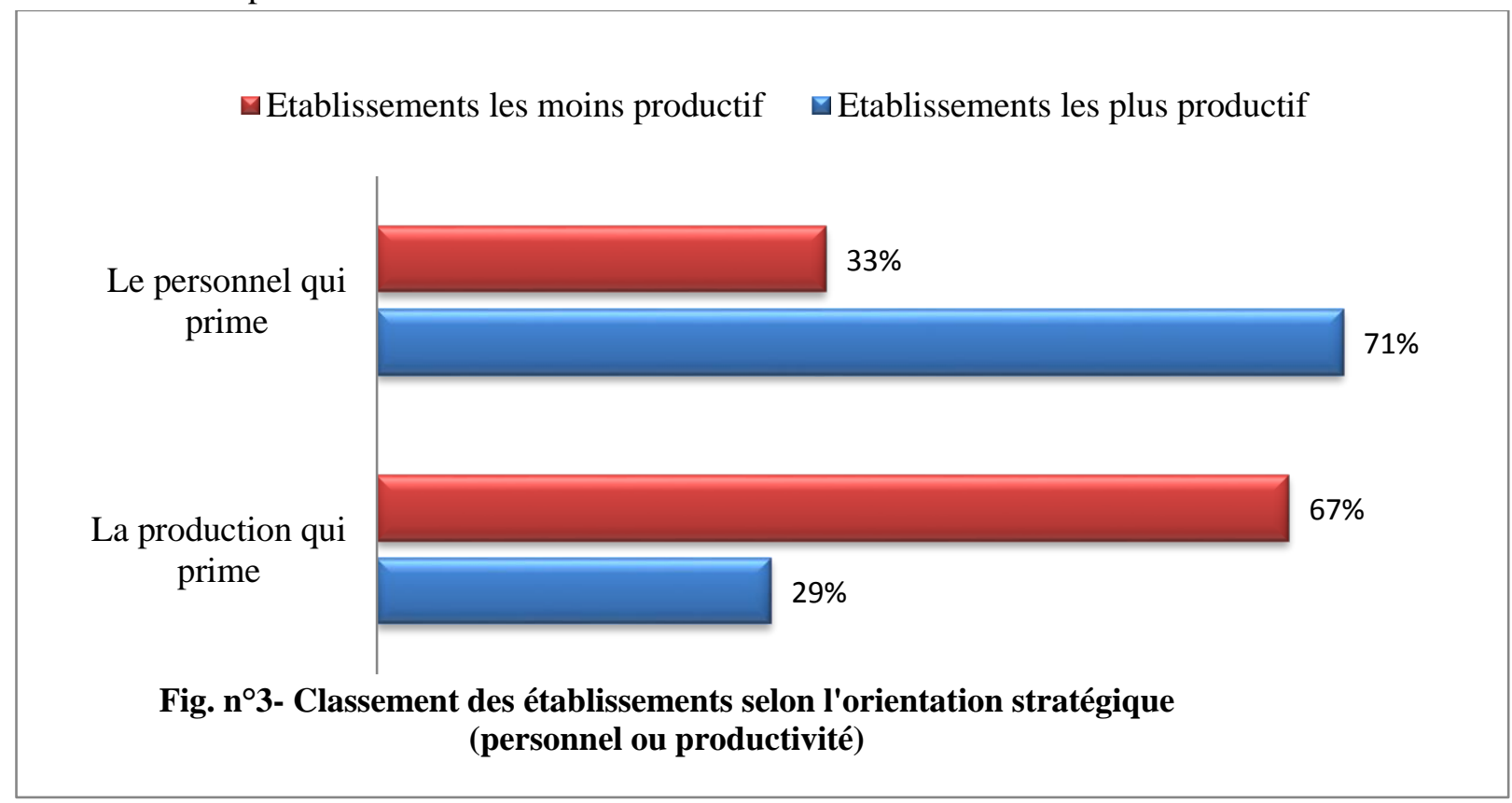

On peut dire, que ce sont les chefs des établissements «soucieux de leur personnel » qui l'emportent. Ces derniers ne se limitent pas à l'amélioration de l'efficacité humaine, par le développement de la motivation et la satisfaction au travail, mais ils le dépassent largement vers une gestion optimale de connaissances et de compétence. Cette gestion s'accomplit par des formations académiques ou professionnelles, en vue d'un développement de carrière suivant la proposition d'un poste meilleur, ou d'un poste de responsabilité, voire même vers un changement de carrière (d'un administrateur vers un professeur).

Comme nous a expliqué un doyen d'un établissement « soucieux de son personnel » : Je m'efforce d'aider le staff administratif à poursuivre leurs études académiques, pour obtenir un diplôme reconnu qui va leur permettre d'obtenir un poste meilleur, d'être promues, ou même de changer de statut... ».

Un autre directeur aussi «soucieux de son personnel» nous a avoué expressément: "j'ai essayé de comprendre et d'étudier le travail de chacun de nos enseignants-chercheurs, en vue de trouver une certaine harmonie et une concordance entre individus, pour pouvoir les regrouper sur le même créneau de travail, voire de les pousser à réaliser des travaux de recherche en commun... ».

À l'encontre, un exemple frappant d'un chef d'établissement « soucieux de la productivité » qui précise que : «Il faut respecter le volume horaire de la part des enseignants et du staff administratif, et être toujours un élément productif quelles que soient les conditions et indépendamment de la situation... »

Un autre chef aussi « soucieux de la productivité » stipule que : «Il n'est pas question d'autoriser le personnel à poursuivre leurs études en plein-temps de travail. Déjà avec un temps aménagé de $9 \mathrm{~h}$ à $16 \mathrm{~h}$ on arrive à peine à gérer et à régler tous les dossiers en instance... ».

Par ailleurs, le style de management participatif qui demeure le style le plus stimulant de la productivité se trouve étroitement lié au développement de la conscience professionnelle et de l'esprit d'appartenance. 
Ce constat a été justifié par une étude de satisfaction menée auprès de la FSJES de Casablanca, où nous avons enregistré une très forte satisfaction de la part des enseignants-chercheurs et du staff administratif à l'égard du style de management participatif adopté par le doyen de cet établissement. À noter que cet établissement demeure au premier rang des établissements les plus productifs suivant cette étude.

Ce chef d'établissement nous a avoué pleinement qu'après sa nomination à la tête de cet établissement, il a constaté un changement radical dans la mentalité de ses collaborateurs, qui sont devenus aussi efficients et s'intéressant davantage à la réputation de l'établissement et à son image de marque, aller même vers à un développement de l'esprit d'appartenance ; «...Cette année, trois de mes collaborateurs ont refusé de se permuter avec d'autres fonctionnaires affectés à leur ville d'origine, pour la simple raison qu'ils ne se voient pas ailleurs».

\section{L'autoritarisme}

Aux pressions exercées en vue de la productivité se rattachent les pratiques de management plus ou moins autoritaires que certains chefs pratiquent. Un management se basant sur une autorité plus étroite correspond généralement à une baisse de productivité, alors qu'un management avec une autorité plus tolérante et plus compréhensive tend à produire un accroissement de celle-ci. Cette corrélation est représentée dans la figure $\mathrm{n}^{\circ} 4$.

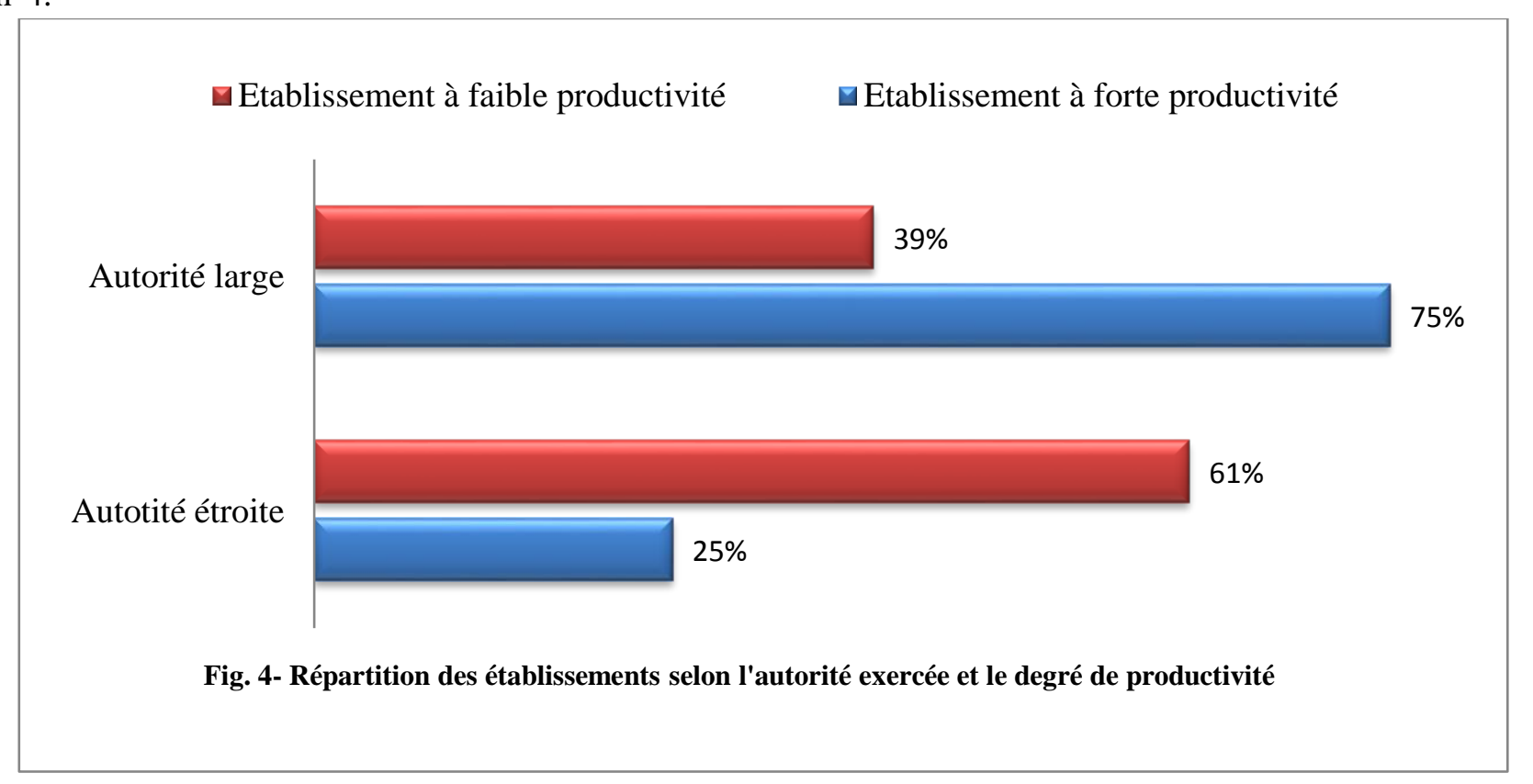

Dans le même ordre d'idée, et dans l'un des établissements étudiés, nous avons constaté qu'avec l'arrivée d'un nouveau chef de département suivant la promotion du premier vers un autre poste de responsabilité a provoqué une baisse de la productivité. Ce constat a été justifié par la plupart d'enseignant interrogé, ayant exprimé leur mécontentement vis-à-vis du style de management adopté par ce nouveau chef de département, ainsi que le désaccord total avec la méthode et l'attitude en matière de pouvoir exercé. Ou comme expliqué un professeur de cet établissement : "Notre département connaissait à l'époque de l'ex chef de département un rendement meilleur puisqu'on a été habitué à une participation dans les décisions à tous les niveaux, et à une participation dans la programmation et l'affectation des cours... Personnellement j'ai été totalement impliqué dans la sphère organisationnelle ». 
Toutefois, nous avons demandé au corps professoral de l'établissement en question, et ayant exprimé une forte satisfaction à l'égard du système d'autorité exercé par l'ex chef de département, de décrire et de classer par ordre d'importance les qualifications professionnelles de ce dernier. Après une analyse détaillée en voici les résultats classés par ordre d'importance :

a. Répartition juste et équitable de travail et description des tâches.

b. Favoriser la communication et veiller au partage d'information.

c. Définir après commun accord les règles et les procédures de travail et les faire respecter.

d. Recevoir, traiter et résoudre les réclamations, et les requêtes.

e. Reconnaitre le travail, et l'effort fourni.

f. Mettre à la disposition de son personnel les outils et les moyens nécessaires pour réussir leur mission.

Aussi, les résultats montrent que, le fait de se soucier uniquement de la réalisation de la charge horaire, et de l'achèvement des formations indépendamment de la qualité de déroulement des cours, de la cadence du travail et de la prise en compte du capital immatériel semble être les causes qui déterminent la régression du niveau de la productivité, tel qu'il a été précisé par un interviewé.

En analysant ces résultats, on découvre que les responsables qui adoptent une autorité large arrivent souvent à obtenir un meilleur rendement de la part de leur collaborateur. Pour la simple raison que ces managers se voient plus :

- Concentré sur la définition des tâches et des missions à réaliser, tout en définissant le chemin critique, les méthodes et les outils les plus appropriés pour y arriver.

- Insistent davantage sur le management par objectif.

- Accordent plus de marge de manœuvre.

Tandis que, les chefs qui appliquent un système d'autorité plus au moins étroit et ayant tendance à ordonner des tâches et suivre le détail de réalisation, n'atteindront pas ou atteindront difficilement les objectifs.

Cela conduit à dire que l'attitude et l'exercice de l'autorité représentent des facteurs causaux de la productivité.

\section{E. La communication organisationnelle}

Il est important de signaler, que nous avons aussi remarqué qu'il existe une très forte relation entre la circulation de l'information, la productivité, et le système d'autorité exercé. En effet, nous avons constaté que le personnel qui bénéficie d'une autorité large partage pleinement l'information avec son supérieur, qui lui aussi, favorise et opte pour la communication et le partage, chose qui participe objectivement à l'amélioration de la productivité, et l'inverse est vrai. Les figures $n^{\circ} 5$ et $\mathrm{N}^{\circ} 6$ justifient ce constat.

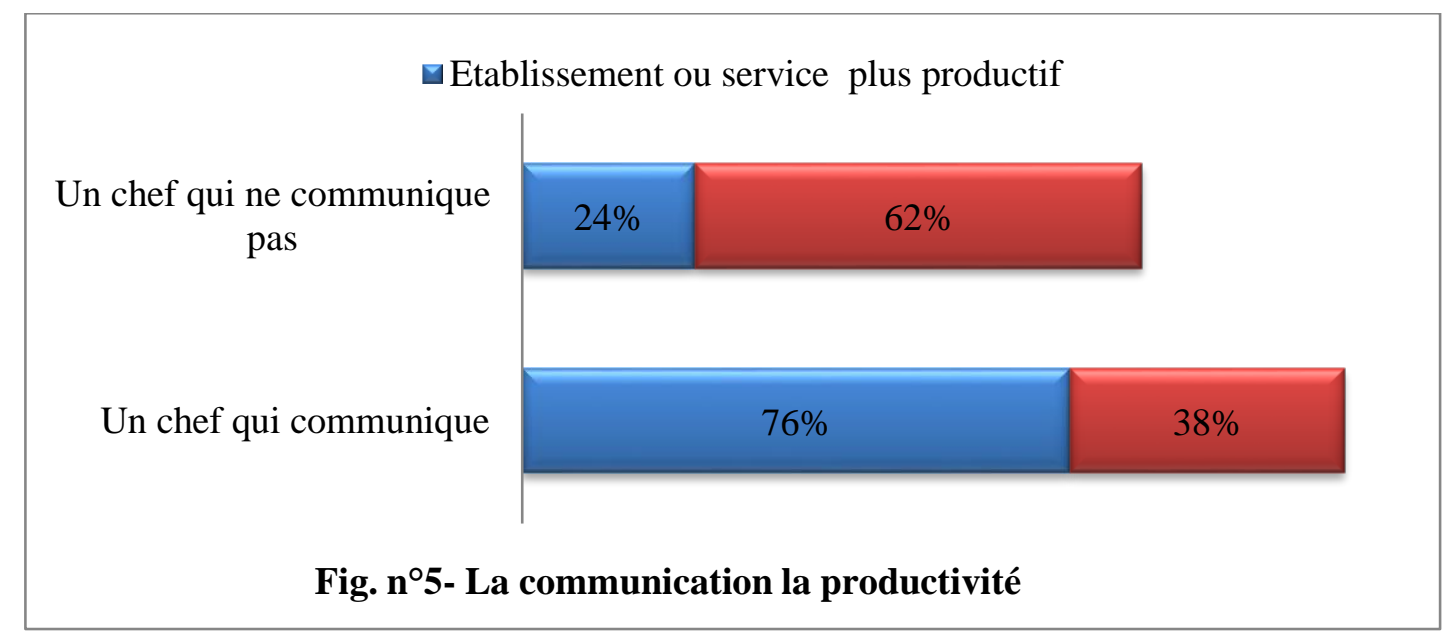




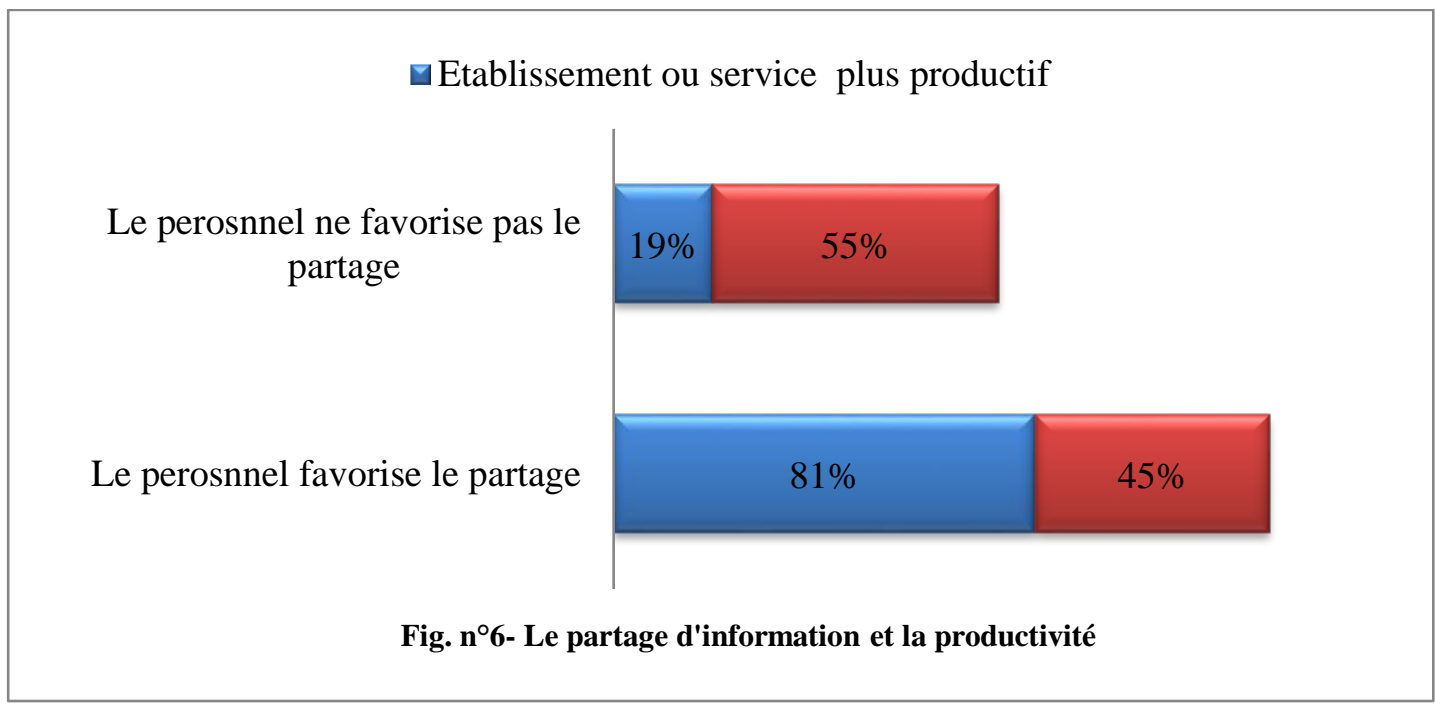

\section{F. La dynamique des groupes et l'esprit d'équipe :}

Le phénomène des groupes qui représente « un système organisé composé d'individus qui partagent des normes, des besoins et des buts, et qui interagissent de manière à influencer mutuellement leur attitude et leur comportement» ${ }^{1}$ a suscité l'intérêt de plusieurs chercheurs, d'autant plus notre étude, du fait qu'il exerce une influence directe sur le développement de l'individu, et surtout qu'il représente un élément extrêmement dynamique de toute organisation sociale, qui influe sur la productivité.

Notre étude nous confirme, que l'aptitude des chefs d'établissement de mettre en place les méthodes les plus appropriées pour une gestion dynamique des groupes en utilisant des explications de phénomènes psychosociaux en termes de forces, de systèmes de tensions et d'équilibre dynamique a tendance à réaliser des taux de productivité et de satisfaction au travail les plus élevés.

Ce constat a été justifié principalement par le lancement en 2008 des réformes de restructuration, de promotion et de valorisation de la recherche scientifique. Ces réformes visaient principalement l'actualisation de la stratégie nationale de la recherche scientifique, la consolidation de son financement, et l'optimisation de la structuration de la recherche scientifique marocaine, et ce, en encourageant la création des laboratoires et des équipes de recherche, ainsi que des centres dédiés à la recherche scientifique, pour plus de productivité et de visibilité.

Plusieurs critères ont été adoptés pour guider la composition de ces équipes de recherches, et ayant contribué favorablement à l'amélioration de la production scientifique, il s'agit à titre indicatif de :

- L'intérêt.

- Le désir réel de participer

- La compétence

- La cohérence et l'homogénéité

- La disponibilité...

${ }^{1}$ Dolan, S.L., Gosselin, E., Carrière, J.Psychologie du travail et comportement organisationnel,(3eéd.), Montréal, éditions Gaëtan Morin, 2007, p. 121. 
À côté du respect de ces principes, il a été adopté certains aspects de fonctionnement des laboratoires de recherche comme :

- L'encadrement des activités.

- Le maintien de la solidarité.

- L'enrichissement du contenu.

La mise en place de cette reconfiguration qui tient compte de la solidarité des groupes a conduit à une promotion et à une évolution considérable de la recherche scientifique marocaine, matérialisée par l'augmentation du nombre et de la qualité des productions et des manifestations scientifiques à tous les niveaux,. La figure $\mathrm{n}^{\circ} 7$ montre clairement cette amélioration au sein de l'université Hassan II.

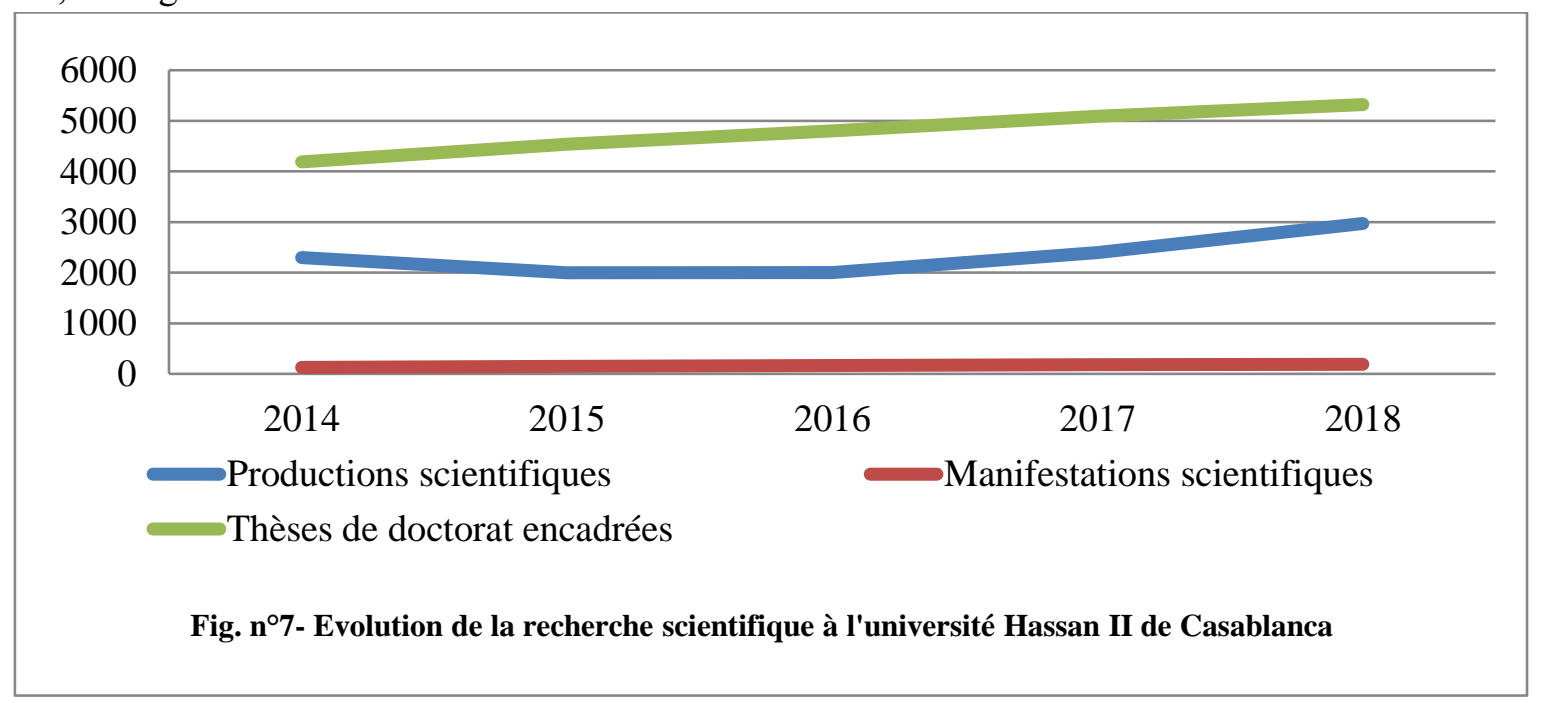

Aussi, notre étude a divulgué une corrélation positive entre le travail en équipe et le degré de productivité. En effet, nous avons découvert que les corps appartenant aux établissements les plus productifs favorisent le travail en équipe, et développent une entraide et une collaboration mutuelle, et ce, par :

- La production d'ouvrages collectifs.

- L'organisation de rencontres scientifiques.

- La production des cours normalisés,

- $\quad \ldots$

$\square$ Avec le travail en équipe $\square$ Contre le travail en équipe

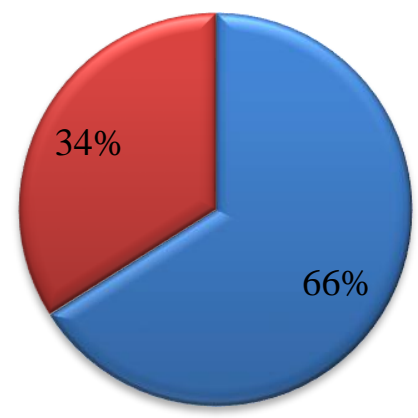

Fig. n $^{\circ}$ - Les corp professoral et administratif les plus productifs 
En outre, et comme l'on peut s'y attendre, les groupes de travail qui favorisent la discussion, et l'échange d'expérience entre eux, et qui sont plus au moins solidaire sont plus efficaces et beaucoup plus productifs que les autres groupes au sein du même établissement ou entre les différents établissements. Figure ${ }^{\circ} 9$.

\section{घL'échange d'expérience Oui घL'échange d'expérience Non}

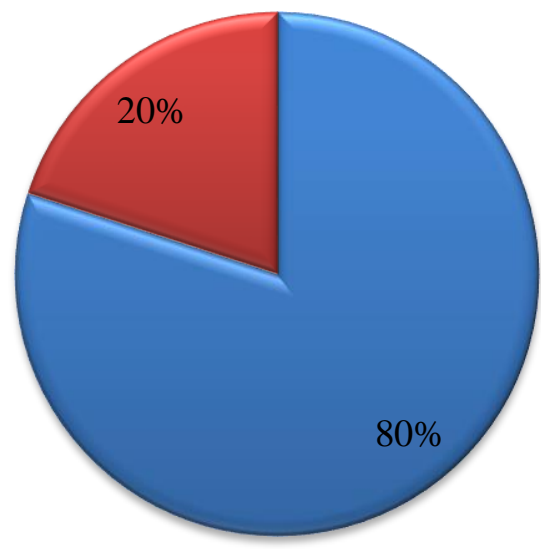

Fig. $\mathbf{n}^{\circ 9}$ - Les équipes des établissements les plus productifs

À préciser aussi que, les groupes à solidarité plus élevée manifestent une tendance à :

- Développer un esprit d'appartenance.

- Développer des relations interpersonnelles entre les collègues.

- Produire plus, mais surtout produire mieux.

Par ailleurs, il semble important de confirmer que le développement de la relation et de l'échange entre les individus demeure en parfaite adéquation avec la productivité. En effet, $64 \%$ des corps professoral et administratif appartenant aux établissements les plus productifs annoncent qu'ils développent des amitiés dans leur groupe de travail plus souvent qu'à l'extérieur. Il divulgue aussi qu'un climat social convivial mène certainement à un rendement meilleur.

Il existe, certes, de nombreux autres déterminants aussi cruciaux pour promouvoir et développer cette notion de dynamique de groupe et d'esprit d'équipe

\section{G. L'autocontrôle}

Un autre facteur contribuant à l'accroissement de la productivité des groupes au sein des établissements les plus productifs est celui de l'autocontrôle. Ce déterminant majeur de la productivité est nettement favorisé par le personnel appartenant aux établissements les plus productifs.

Cette étude a montré que le corps professoral ou même le corps administratif les plus productifs ont tendance a favorisé l'autocontrôle de leur réalisation, plutôt que de subir un contrôle actif ou réactif traditionnellement accompli par les dirigeants.

Les résultats de cette recherche associés à d'autres études révèlent que ce sont principalement le style et le mode de management, la structure, les procédures et les systèmes qui déterminent cette dynamique de groupe. 
Parmi ces dernières, notons la participation du groupe aux décisions et aux résultats, ainsi que l'implication et l'engagement des individus dans la sphère organisationnelle.

\section{CONCLUSION}

Les différents résultats présentés permettent alors de tirer une conclusion importante concernant les principes fondamentaux de motivation au travail. L'examen, tant des résultats exposés que de ceux qui ont été obtenus ailleurs, prouve que pour définir un schéma d'évolution organisationnel, faut intégrer le management interculturel comme étant un maillon important du principe de motivation au travail. Un management qui admet le rapprochement entre plusieurs expertises et techniques, et surtout entre des traits personnels divers, et qui privilégiait la dimension humaine dans son analyse organisationnelle, en se référant à la communication interpersonnelle et aux échanges sociaux.

Bien que ces conclusions restent particulières et propres au contexte d'investigation auquel nous nous sommes intéressés, il n'en demeure pas moins, qu'elles restent modulables selon le contexte, la vision et la coalition de chacun.

En effet, nous avons cherché à travers cette étude à présenter les principes généraux de la motivation au travail que les établissements de l'enseignement supérieur marocains peuvent les prendre en considération, en guise d'une amélioration du fonctionnement organisationnel. Pour y aboutir, ces principes doivent se baser sur l'élément humain et la culture organisationnelle comme déterminants majeurs de la productivité.

Nous pensons alors que les résultats de cette étude même s'ils ne sont pas généralisables à toute action de restructuration, ils pourront servir comme référence en termes de leviers d'action à optimiser, des modalités à adopter, et de dimensions organisationnelles et structurelles à prendre en considération dans l'amorçage et la mise en exergue de tout changement.

En outre, les résultats produits par notre étude au sein des établissements de l'enseignement supérieur marocains peuvent être exploités directement par toutes organisations opérantes dans le secteur de l'enseignement supérieur marocain et désirant optimiser leurs actions de restructuration et reconfiguration escomptées, comme elles représenteront un point de réflexion ultérieur pour d'autres investigations.

\section{BIBLIOGRAPHIE}

[1] Allard-Poesi F., (2017) ; « La construction du sens, l'organisation et le manager : les apports de Karl Weick», IRG- Institut de recherche en gestion.

[2] Alter N., (2001) ; «L'innovation ordinaire », Paris, Presses Universitaires de France, 2001.

[3] Autissier D. \& Moutot JM, (2016); “ Méthodes de conduite du changement : Diagnostic, Accompagnement, Performance » DUNOS, 4ème édition.

[4] Bartoli, (2009) ; « Management dans les organisations publiques ». Edition Dunod, P210 et suite.

[5] Ben Abdallah L. \& Ben Ammar Mamlouk Z., (2007) ; «Changement organisationnel et évolution des compétences » La Revue des Sciences de Gestion, 2007/4 (n²26-227).

[6] Benaissa H., (2001) ; «Quelle méthodologie de recherche appropriée pour une construction de la recherche en gestion », Xième conférence de l'association internationale de management stratégique 13-14-15 juin 2001, p15-16.

[7] Brown S.L., Eisenhardt K.M., (2007); "The Art of Continuous Change: Linking Complexity Theory and Time-paced Evolution in Relentlessly Shifting Organizations", Administrative Science Quarterly, vol. 42, p. 1-34.

[8] Child J., (1972) ; “Organizational structure, environment and performance : the role of strategic choice”, Sociology, Vol 6, N¹.

[9] David A., Laurent G., Kevin J.J \& Moutot JM., (2013) : “étude portant sur les liens entre les caractéristiques et le succès des changements organisationnels » publiée en Question (s) de management 2013/2 N³.

[10] Dolan, S.L., Gosselin, E., Carrière, J.Psychologie du travail et comportement organisationnel,(3eéd.), Montréal, éditions Gaëtan Morin, 2007, p. 121. 
[11] Drucker P., (2012) ; « L’avenir du management : Réflexion pour l'action », Paris, Edition Pearson, p184.

[12] Faraji A., (2015); “ La gestion entrepreneuriale du secteur public pour une nouvelle approche managériale » Revue du droit marocain, $\mathrm{N}^{\circ} 2$, p359.

[13] Gérard M., Rangeon F. \& Thibault J-L., (1997) ; « Les relations contractuelles entre collectivités publiques», dans Godard, Francis, Le gouvernement des villes. Territoire et pouvoir, Paris, Descartes \& Cie, p. 140.

[14] Ghouati A., (2010); “L’enseignement supérieur au Maroc : de l’autonomie à la dépendance ? », JHES/RESA Vol. 8, №1, 2010 .

[15] Komat A., (2007); "Analyse des pratiques de gestion des ressources humaines dans les entreprises industirelles marocaines: état des lieux face aux performance imposes par le processus d'ouverture".

[16] Miles M-B. \& Huberman AM., (2003) ; “Analyse des données qualitatives ». TradRispal M-H., Edition De boeck. p58.Nadler D., (1994); "Beyond the Heroic leader", in Discontinous Change: leading Organizational Transformation, by Nadler D, Schaw R, \& Walton E, Jossey-Bass Publishers, San francisco, pp217-231.

[17] Rapport mondial NO. 43173-MNA, Juillet 2008.

[18] Rapport Reflecting on Peace Practice ; pour consulter la liste complète des théories de changement se rendre à Annexe 6. Théories du changement Revue de l'OCDE sur le développement 2008/3 (n ${ }^{\circ}$ ) p44.

[19] Rapport de recherche sur Les réformes des systèmes de gouvernance dans l'enseignement supérieur au Maroc. Kouhlani. B, Ennaji M.M.

[20] Relative productivity of university technology transfer offices: an exploratory study. Res. Policy 32 (1), 27-48.

[21] Siegel, D.S., Waldman, D., Link, A, (2003); “Assessing the impact of organizational practices".

[22] Tarhi MA., (2018); "La conduite du changement au sein des EES marocains : construction d'un modèle de changement organisationnel contextualisé à l'aide de la recherche intervention ».

[23] Vas A., (2001); “Top Management skills in a context of endemic organizational change: The Case of Belgacom”, Journal of General Management, vol. 27, $n^{\circ} 1$, p. 71-89. 